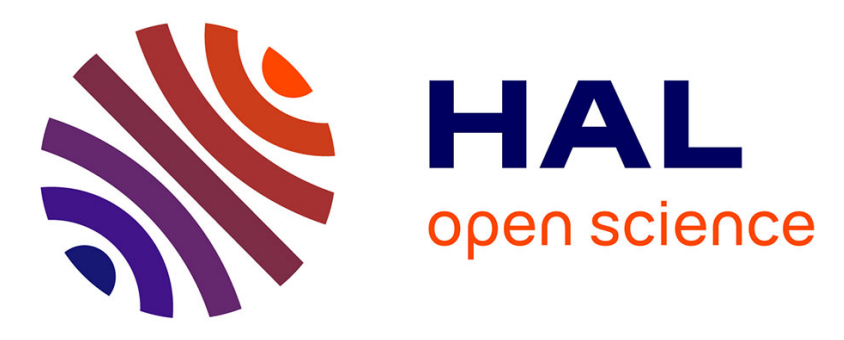

\title{
Exponential Stability Analysis of Sampled-data ODE-PDE Systems and Application to Observer Design
}

\author{
Tarek Ahmed-Ali, Iasson Karafyllis, Fouad Giri, Miroslav Krstic, Françoise
}

Lamnabhi-Lagarrigue

\section{- To cite this version:}

Tarek Ahmed-Ali, Iasson Karafyllis, Fouad Giri, Miroslav Krstic, Françoise Lamnabhi-Lagarrigue. Exponential Stability Analysis of Sampled-data ODE-PDE Systems and Application to Observer Design. 22nd International Symposium on Mathematical Theory of Networks and Systems (MTNS), Jul 2016, Minneapolis, United States. hal-01313774

\section{HAL Id: hal-01313774 \\ https://hal-centralesupelec.archives-ouvertes.fr/hal-01313774}

Submitted on 13 Jul 2020

HAL is a multi-disciplinary open access archive for the deposit and dissemination of scientific research documents, whether they are published or not. The documents may come from teaching and research institutions in France or abroad, or from public or private research centers.
L'archive ouverte pluridisciplinaire HAL, est destinée au dépôt et à la diffusion de documents scientifiques de niveau recherche, publiés ou non, émanant des établissements d'enseignement et de recherche français ou étrangers, des laboratoires publics ou privés. 


\title{
Exponential Stability Analysis of Sampled-Data ODE-PDE Systems and Application to Observer Design
}

\author{
Tarek Ahmed-Ali, Iasson Karafyllis, Fouad Giri, Miroslav Krstic, and F. Lamnabhi-Lagarrigue
}

\begin{abstract}
A small-gain approach is presented for analyzing exponential stability of a class of (dynamical) hybrid systems. The systems considered in the paper are composed of finite-dimensional dynamics, represented by a linear Ordinary Differential Equation (ODE), and infinite-dimensional dynamics described by a parabolic Partial Differential Equation (PDE). Exponential stability is established under conditions involving the maximum allowable sampling period (MASP). This new stability result is shown to be useful in the design of sampled-output exponentially convergent observers for linear systems that are described by an ODE-PDE cascade. The new stability result also proves to be useful in designing practical approximate observers involving no PDEs.
\end{abstract}

Index Terms-ODE-PDE cascade systems, sampled-data systems, observer design, backstepping approach, exponentially convergent observers.

\section{INTRODUCTION}

A great deal of interest has been paid to designing sampledoutput exponentially convergent observers for finite-dimensional continuous-time systems described by nonlinear ODEs. Various approaches have been proposed that differ from each other in the way data-sampling is accounted for in the observer design and analysis, see e.g. $[1,2,3,9,10]$. Considerably less effort has been devoted to the problem of designing sampled-output observers for systems involving PDEs. In [4], an observer with zero-orderhold $(\mathrm{ZOH})$ sampled innovation term has been proposed for a class of semi-linear systems described by a scalar diffusion equation. Using Lyapunov-Krasovskii functional, sufficient conditions for exponential stability have been emphasized in terms of Linear matrix Inequalities (LMIs) allowing the determination of the observer gain and sampling interval. In [5], a sampled-output observer featuring time-varying gain has been proposed for a class of semilinear systems described by parabolic PDEs. The time-varying gain was shown to be beneficial to achieve larger MASPs. Again, Lyapunov-Krasovskii functional and LMIs have been resorted to establish the observer exponential stability and characterizing the MASP.

This paper is focused on cascade systems introduced in [6], composed of an ODE connected in series with a PDE of parabolic type, which might account for diffusion sensor dynamics. We seek the development of an observer that is able to provide accurate online estimates of both the ODE-subsystem state and the PDE-subsystem state, making use of sampled output measurements. In the case where continuous-time output measurements are available, exponentially convergent observers are obtained using the backstepping design approach developed in [6]. Key design aspects of this approach include Volterra-type state transformations and a Lyapunov functional. These tools

T. Ahmed-Ali and F. Giri are with GREYC Lab, University of Caen, ENSICAEN, 14032 Caen, France (Tarek.Ahmed-Ali@ensicaen.fr, fouad.giri@unicaen.fr).

I. Karafyllis is with the Department of Mathematics, National Technical University of Athens, Athens, Greece, (e-mail: iasonkar@ central.ntua.gr).

M. Krstic is with the Department of Mechanical and Aerospace Engineering, University of California at San Diego, La Jolla, CA 92093-0411, USA (e-mail: krstic@ucsd.edu).

F. Lamnabhi-Lagarrigue, is with LSS-CNRS, SUPELEC, EECI, 91192 Gifsur-Yvette, France (email: Francoise.Lamnabhi-Lagarrigue@1ss.supelec.fr). have proved to be useful in continuous-time observer design for systems that are (entirely or partially) described by PDEs.

Inspired by the above approach, a new design method is presently developed to get exponentially convergent observers in the case where only sampled-output measurements are available. The new design approach is built on a stand-alone exponentialstability result that we present for a class of hybrid systems consisting of an autonomous parabolic PDE connected with an ODE through a ZOH-sampler set. The stability analysis is performed making use of the small-gain technique. A second contribution of this study consists in developing a sampledoutput, version of the backstepping-based observer design of [6] and showing that the resulting error system fits the class of hybrid systems that is analyzed in the aforementioned technical stability result. The observer design is based on emulation principles. Invoking this proposition, we get sufficient conditions for the observer to be exponentially stable. Interestingly, the sufficient conditions allow an explicit determination of the MASP. Another contribution of this work is the development of an ODE-based approximation of the above sampled-output observer. Since it is only defined by ODEs, the approximate observer will prove to be more suitable for practical use. Interestingly, the approximate observer accuracy is also formally evaluated using the preliminary technical stability result. A part of the above contributions, including those in Sections 2 and 3, will be presented in the 2016 MTNS conference [12].

The paper is organised as follows: in Section 2, the new standalone technical stability result for hybrid systems is established; the sampled-output observer problem for ODE-PDE systems is formulated and dealt with in Section 3; an ODE-based approximation of the sampled-output observer is developed in Section 4; a conclusion and reference list end the paper.

Notation. The $n$ dimensional real space is denoted $\mathbf{R}^{n}$ and the corresponding Euclidean norm is denoted $\| . \mathbf{R}^{n \times m}$ is the set of all $n \times m$ real matrices and || designates matrix norm induced by vector Euclidian norm. The continuous-time is denoted $t$ while $\left\{t_{k}\right\}_{k=0}^{\infty}$ refers to any real increasing time sequence such that $t_{0}=0, \lim _{k \rightarrow \infty} t_{k}=+\infty$, and $\sup _{k \geq 0}\left(t_{k+1}-t_{k}\right)=T$, for some $0<T<\infty$. A time sequence $\left\{t_{k}\right\}_{k=0}^{\infty}$ with these properties is called a partition of $\mathbf{R}_{+}$and the associated smallest real constant $T$ is its diameter. $C^{k}(E, F)$ denotes the set of functions, from some set $E$ to some $F$, that are $k$ times continuously differentiable (for some $k \in \mathbf{N}$ ). The $L^{2}$-norm of a function defined on the interval $[0,1]$ is denoted $\|\cdot\|$ and $L^{2}[0,1]$ is the Hilbert space of square integrable functions. Accordingly, $\eta \in L^{2}[0,1] \Leftrightarrow\|\eta\| \stackrel{\text { def }}{=}\left(\int_{0}^{1}|\eta(\varsigma)|^{2} d \varsigma\right)^{1 / 2}<\infty . L_{l o c}^{\infty}\left(\mathbf{R}_{+} ; \mathbf{R}^{m}\right)$ denotes the space of measurable and locally essentially bounded functions $\quad \eta: \mathbf{R}_{+} \rightarrow \mathbf{R}^{m}$. Given a function $w:[0,1] \times \mathbf{R}_{+} \rightarrow \mathbf{R} ;(x, t) \rightarrow w(x, t)$, the notations $w_{x}(x, t)$ and $w_{t}(x, t)$ refer to its partial derivatives while $w[t]$ and $w_{x}[t]$ 
refer to the functions defined on $0 \leq x \leq 1$ by $(w[t])(x)=w(x, t)$ and $\left(w_{x}[t]\right)(x)=\partial w(x, t) / \partial x$.

\section{Preliminary Stability Result FOR a Class of Hybrid SYSTEMS}

In this section, we analyze the class of systems composed of a parameter distributed subsystem and a finite-dimensional subsystem interacting as follows:

$$
\begin{aligned}
& \dot{X}(t)=A_{0} X(t)+A_{1} X\left(t_{k}\right)+b w\left(0, t_{k}\right)+G z(t), \\
& \text { for all } t \in\left[t_{k}, t_{k+1}\right) \text { a.e. and all integers } k \geq 0, \\
& w_{t}(x, t)=w_{x x}(x, t), \text { for }(x, t) \in(0,1) \times(0,+\infty), \text { a.e. }
\end{aligned}
$$

with boundary conditions,

$w_{x}(0, t)=0$ and $w(1, t)=0$, for all $t \geq 0$

with initial conditions,

$$
X(0)=X_{0} \text { and } w(x, 0)=(w[0])(x) \text { for } x \in[0,1]
$$

where $X(t) \in \mathbf{R}^{n}$ denotes a finite-dimensional state vector, $w(x, t) \in \mathbf{R}$ a distributed state variable, and $z(t) \in \mathbf{R}^{m}$ is its external signal that is measurable and locally essentially bounded; $A_{0}, A_{1} \in \mathbf{R}^{n \times n}$ and $G \in \mathbf{R}^{n \times m}$ are constant matrices and $b \in \mathbf{R}^{n}$ is vector; $\left\{t_{k}\right\}_{k=0}^{\infty}$ is a partition of $\mathbf{R}_{+}$. It is seen that the subsystem represented by the linear parabolic PDE (2)-(3), is continuous-time, autonomous and acts on the finite-dimensional subsystem represented by the linear ODE (1), through a ZOHsampler leading to a hybrid system.

The system described by (1), (2) and (3) results from the cascade connection of the infinite-dimensional system (2), (3) with the finite-dimensional hybrid system

$$
\dot{X}(t)=A_{0} X(t)+A_{1} X\left(t_{k}\right)+z_{a}(t)
$$

where

$$
z_{a}(t):=b w\left(0, t_{k}\right)+G z(t), \text { for all } t \in\left[t_{k}, t_{k+1}\right) \text { and } k \geq 0
$$

For the infinite-dimensional system (2), (3), we have the following lemma, whose proof can be found in Appendix A.

Lemma 1. For every $w[0] \in C^{2}[0,1]$ with $(w[0])(1)=\left(w_{x}[0]\right)(0)=0$, the initial value problem (2), (3) has a unique solution $w[t] \in C^{2}[0,1]$ defined for all $t \geq 0$, which satisfies the following inequalities, for all $t \geq 0$ :

$$
\|w[t]\| \leq \exp \left(-\frac{\pi^{2}}{4} t\right)\|w[0]\|,\left\|w_{x}[t]\right\| \leq \exp \left(-\frac{\pi^{2}}{4} t\right)\left\|w_{x}[0]\right\|
$$

The Input-to-State Stability (ISS) property of the finitedimensional hybrid system (5) is established in the following lemma.

Lemma 2. Consider the hybrid system (5) and suppose the matrix $A_{0}+A_{1}$ is Hurwitz. Let $R, \lambda>0$ be any real constants and $\phi: \mathbf{R}_{+} \rightarrow \mathbf{R}_{+}$be any continuous function satisfying, for all $t \geq 0$ :

$$
\left|\exp \left(\left(A_{0}+A_{1}\right) t\right)\right| \leq R \exp (-\lambda t) \text { and }\left|\exp \left(A_{0} t\right)\right| \leq \phi(t)
$$

Also, let $T>0$ and $\sigma \in(0, \lambda)$ be any real constants satisfying:

$$
\lambda>\sigma+R\left|A_{1}\right| \exp (\sigma T)\left(\left|A_{0}\right|+\left|A_{1}\right|\right) \int_{0}^{T} \phi(s) d s
$$

Then, there exist real constants $\bar{K}, \bar{\gamma}>0$ such that for every $z_{a} \in L_{l o c}^{\infty}\left(\mathbf{R}_{+} ; \mathbf{R}^{m}\right), \quad X_{0} \in \mathbf{R}^{n}$, and any $T$-diameter partition $\left\{t_{k}\right\}_{k=0}^{\infty}$ of $\mathbf{R}_{+}$, the unique solution of the initial value problem
(5) with $X(0)=X_{0}$ exists for all $t \geq 0$ and satisfies the following inequality, for all $t \geq 0$ :

$$
|X(t)| \leq \bar{K} \exp (-\sigma)\left|X_{0}\right|+\bar{\gamma} \sup _{0 \leq s \leq t}\left(\left|z_{a}(s)\right| \exp (-\sigma(t-s))\right) \text {. }
$$

The proof of Lemma 2 is performed by means of a small-gain argument, which gives the sufficient small-gain condition (9).

Proof. Existence and uniqueness of the initial value problem (5) with $X(0)=X_{0}$ is a direct consequence of the step-by-step construction of the solution of (5) in each interval $\left[t_{k}, t_{k+1}\right]$.

To establish (10), introduce the following auxiliary variables:

$$
q(t):=t_{k} \text {, for all } t \in\left[t_{k}, t_{k+1}\right) \text { and all integers } k \geq 0
$$

On the other hand, using definition (11), equation (5) rewrites $\dot{X}(t)=\left(A_{0}+A_{1}\right) X(t)+A_{1}(X(q(t))-X(t))+z_{a}(t)$, with initial condition $X(0)=X_{0}$. It follows that the solution of (5) satisfies the following equation:

$$
\begin{aligned}
X(t)= & \exp \left(\left(A_{0}+A_{1}\right) t\right) X_{0} \\
& +\int_{0}^{t} \exp \left(\left(A_{0}+A_{1}\right)(t-s)\right) A_{1}(X(q(s))-X(s)) d s \\
& +\int_{0}^{t} \exp \left(\left(A_{0}+A_{1}\right)(t-s)\right) z_{a}(s) d s
\end{aligned}
$$

Since $\sigma \in(0, \lambda)$, one gets from (8) and (12) that, for all $t \geq 0$ :

$$
\begin{aligned}
\sup _{0 \leq s \leq t}(|X(s)| \exp (\sigma s)) & \leq R\left|X_{0}\right|+\frac{R}{\lambda-\sigma} \sup _{0 \leq s \leq t}\left(\left|z_{a}(s)\right| \exp (\sigma s)\right) \\
& +\frac{R\left|A_{1}\right|}{\lambda-\sigma} \sup _{0 \leq s \leq t}(|X(q(s))-X(s)| \exp (\sigma s))
\end{aligned}
$$

By (8), one has $\left|\exp \left(A_{0} t\right)-I\right| \leq\left|A_{0}\right| \int_{0}^{t} \phi(s) d s$ for all $t \geq 0$. Also, applying the variation of constants formula one gets, using (5):

$$
\begin{aligned}
X(t)= & \exp \left(A_{0}\left(t-t_{k}\right)\right) X\left(t_{k}\right)+\int_{t_{k}}^{t} \exp \left(A_{0}(t-s)\right) A_{1} X\left(t_{k}\right) d s \\
& +\int_{t_{k}}^{t} \exp \left(A_{0}(t-s)\right) b z_{a}(s) d s
\end{aligned}
$$

for all $t \in\left[t_{k}, t_{k+1}\right)$ and all integers $k \geq 0$. From the above facts it follows that, for all $t \in\left[t_{k}, t_{k+1}\right)$ and all integers $k \geq 0$ :

$$
\begin{aligned}
& \left|X(t)-X\left(t_{k}\right)\right| \leq\left(\left|A_{0}\right|+\left|A_{1}\right|\right) \int_{0}^{t-t_{k}} \phi(s) d s\left|X\left(t_{k}\right)\right| \\
& +\exp (-\sigma) \int_{0}^{t-t_{k}} \phi(s) \exp (\sigma s) d s \sup _{t_{k} \leq s \leq t}\left(\left|z_{a}(s)\right| \exp (\sigma s)\right)
\end{aligned}
$$

Using the inequality $\sup _{k \geq 0}\left(t_{k+1}-t_{k}\right) \leq T$ and definition (11), it follows from (14) that, for all $t \geq 0$ :

$$
\begin{aligned}
& \sup _{0 \leq s \leq t}(|X(s)-X(q(s))| \exp (\sigma s)) \\
& \leq \exp (\sigma T)\left(\left|A_{0}\right|+\left|A_{1}\right|\right) \int_{0}^{T} \phi(s) d s \sup _{0 \leq s \leq t}(|X(s)| \exp (\sigma s)) \\
& \quad+\int_{0}^{T} \phi(s) \exp (\sigma s) d s \sup _{0 \leq s \leq t}\left(\left|z_{a}(s)\right| \exp (\sigma s)\right)
\end{aligned}
$$

Combining (13) and (15), one gets for all $t \geq 0$ : $\sup _{0 \leq s \leq t}(|X(s)| \exp (\sigma s)) \leq R|X(0)|$

$$
+\frac{R\left|A_{1}\right|}{\lambda-\sigma} \exp (\sigma T)\left(\left|A_{0}\right|+\left|A_{1}\right|\right) \int_{0}^{T} \phi(s) d s \sup _{0 \leq s \leq t}(|X(s)| \exp (\sigma s))
$$




$$
+\frac{R}{\lambda-\sigma}\left(1+\left|A_{1}\right| \int_{0}^{T} \phi(s) \exp (\sigma s) d s\right) \sup _{0 \leq s \leq t}\left(\left|z_{a}(s)\right| \exp (\sigma s)\right)
$$

Using (9), inequality (16) yields:

$$
\begin{aligned}
& \left.\sup _{0 \leq s \leq t}|X(s)| \exp (\sigma s)\right) \\
& \leq R \frac{(\lambda-\sigma)|X(0)|+\left(1+\left|A_{1}\right| \int_{0}^{T} \phi(s) \exp (\sigma s) d s\right) \sup _{0 \leq s \leq t}\left(\left|z_{a}(s)\right| \exp (\sigma s)\right)}{\lambda-\sigma-R\left|A_{1}\right| \exp (\sigma T)\left(\left|A_{0}\right|+\left|A_{1}\right|\right)_{0}^{T} \phi(s) d s}
\end{aligned}
$$

which establishes (10) for certain constants $\frac{0}{K}, \bar{\gamma}>0$. The proof of Lemma 2 is complete. $\triangleleft$

The ISS property of the system (1)-(3) is described in Proposition 1, which constitutes an instrumental tool in the subsequent observer design.

Proposition 1. Consider the hybrid system (1)-(3) and suppose the matrix $A_{0}+A_{1}$ is Hurwitz. Let $R, \lambda>0$ be any real constants and $\phi: \mathbf{R}_{+} \rightarrow \mathbf{R}_{+}$be any continuous function satisfying (8) for all $t \geq 0$. Also, let $T>0$ and $\sigma \in(0, \lambda) \cap\left(0, \pi^{2} / 4\right]$ be any real constants satisfying (9). Then, there exist real constants $K, \gamma>0$ such that for every $z \in L_{\text {loc }}^{\infty}\left(\mathbf{R}_{+} ; \mathbf{R}^{m}\right), \quad X_{0} \in \mathbf{R}^{n}, \quad w[0] \in C^{2}[0,1] \quad$ with $(w[0])(1)=\left(w_{x}[0]\right)(0)=0$, and any $T$-diameter partition $\left\{t_{k}\right\}_{k=0}^{\infty}$ of $\mathbf{R}_{+}$, the unique solution of the initial value problem (1)-(4) exists for all $t \geq 0$ and satisfies the following inequalities for all $t \geq 0$ :

$$
|X(t)| \leq K \exp (-\sigma)\left(\left|X_{0}\right|+\left\|w_{x}[0]\right\|\right)+\gamma \sup _{0 \leq s \leq t}(|z(s)|),
$$

Proof. By virtue of (3) one has $w(0, t)=-\int_{0}^{1} w_{x}(s, t) d s$ for all $t \geq 0$. Using the Cauchy-Schwarz inequality, one gets for all $t \geq 0$ and $x \in[0,1]$ :

$$
|w(0, t)|=\left|\int_{0}^{1} w_{x}(s, t) d s\right| \leq \int_{0}^{1}\left|w_{x}(s, t)\right| d s \leq\left\|w_{x}[t]\right\|
$$

for all $t \geq 0$, which together with the second inequality in (7) gives:

$$
\left|w\left(0, t_{k}\right)\right| \leq \exp \left(-\frac{\pi^{2}}{4} t_{k}\right)\left\|w_{x}[0]\right\|, \text { for all integers } k \geq 0
$$

Using (19) and the fact that $\sigma \leq \pi^{2} / 4$ and $\sup _{k \geq 0}\left(t_{k+1}-t_{k}\right) \leq T$, it follows from (6) that:

$$
\begin{aligned}
& \sup _{0 \leq s \leq t}\left(\left|z_{a}(s)\right| \exp (\sigma s)\right) \leq|b| \sup _{\substack{t_{k} \leq s<t_{k+1} \\
k \in \mathbf{N}}} \exp \left(\sigma s-\frac{\pi^{2}}{4} t_{k}\right)\left\|w_{x}[0]\right\| \\
& +|G| \exp (\sigma) \sup _{0 \leq s \leq t}(|z(s)|) \\
& \leq|b| \sup _{\substack{t_{k} \leq s<t_{k+1} \\
k \in \mathbf{N}}} \exp \left(\sigma\left(t_{k+1}-t_{k}\right)\right)|| w_{x}[0]|+| G \mid \exp (\sigma) \sup _{0 \leq s \leq t}(|z(s)|) \\
& \leq|b| \exp (\sigma T)|| w_{x}[0]|+| G \mid \exp (\sigma) \sup _{0 \leq s \leq t}(|z(s)|)
\end{aligned}
$$

Inequality (17) with appropriate constants $K, \gamma>0$ is a direct consequence of estimate (10) and inequality (20). The proof of Proposition 1 is complete. $\triangleleft$

Remark 1. Inequalities (17) and (7) guarantee that the overall infinite-dimensional hybrid system (1)-(3) satisfies the ISS property with respect to the input $z$ with linear gain and exponentially decaying effect of the initial conditions. The reader can also see [11] for the ISS property for general infinitedimensional systems. $\triangleleft$

\section{SAMPLED-OUtPUT OBSERVER DESIGN FOR ODE-PDE CASCADES}

\section{A. Class of observed systems}

In this section, we are interested in a class of continuous-time systems assuming the following ODE-PDE cascade structure:

$$
\begin{aligned}
& \dot{X}(t)=A X(t)+B v(t), \text { for } t \geq 0 \\
& u_{t}(x, t)=u_{x x}(x, t)+g(x, v(t)), \\
& \quad \text { for }(x, t) \in(0,1) \times(0,+\infty) \text { a.e. } \\
& u_{x}(0, t)=0, \text { for all } t \geq 0 \\
& u(1, t)=C X(t), \text { for all } t \geq 0
\end{aligned}
$$

with $X(0)=X_{0}$ where $X(t) \in \mathbf{R}^{n}$ denotes the state vector of the finite-dimensional subsystem described (21), and $v(t) \in \mathbf{R}^{m}$ is an external input signal of class $C^{1}\left(\mathbf{R}_{+} ; \mathbf{R}^{m}\right) ; \quad u(x, t) \in \mathbf{R}$ is the state of the infinite-dimensional subsystem described by the parabolic type PDE (22) with boundary conditions (23)-(24). The quantities $A \in \mathbf{R}^{n \times n}, B \in \mathbf{R}^{n \times m}, C \in \mathbf{R}^{1 \times n}$ are constant matrices and $g(x, v)$ is a function of class $C^{1}\left([0,1] \times \mathbf{R}^{m} ; \mathbf{R}\right)$. The pair $(A, C)$ is observable and the whole system is observed through $\mathrm{ZOH}$ sampling of the signal $u(0, t)$, i.e. the system output is:

$$
y(t)=u\left(0, t_{k}\right) \text {, for all } t \in\left[t_{k}, t_{k+1}\right) \text { and } k \geq 0
$$

where $\left\{t_{k}\right\}_{k=0}^{\infty}$ denotes the sampling time sequence, supposed to be a partition of $\mathbf{R}_{+}$with diameter $T$. We seek an observer that provides accurate online estimates of both the (finitedimensional) state vector $X(t)$ and the distributed state $u(x, t)$, $0 \leq x \leq 1$, based the system input $v(t)$ and the output $y(t)$. The signal $u(1, t)$ is not accessible to measurements.

Remark 2. An illustrating example of the infinite-dimensional subsystem (22)-(24) is shown by Fig. 1. The LTI subsystem is a PWM DC-DC static power converter of the buck type supplying a heating resistor. The back converter is well known to be modelled by a second-order linear ODE, e.g. [13], and its control input is $v_{1}(t)$ is the duty ratio function. The generated heat is diffused along a bar, with $u(1, t)$ and $u(0, t)$ denoting the temperatures at the bar extremities and $g(x, v)$ reflecting the effect of the ambient temperature, denoted $v_{2}(t)$, at a position $x$ along the bar. In this setting, the external input signal is $v(t)=\left[v_{1}(t) v_{2}(t)\right]^{T} \cdot \triangleleft$

Remark 3. In the case where the continuous-time output $u(0, t)=C X(t)$ is accessible to measurements and $g(x, v) \equiv 0$, continuous-time exponentially stable observers are obtained using the backstepping-like design method developed in [6]. An extension of this observer design has been developed in [14] for ODE-PDE systems involving a Lipschitz nonlinearity in the ODE while $g(x, v) \equiv 0$. Presently, a sampled-output version of the observer of [6] will be developed. $\triangleleft$ 


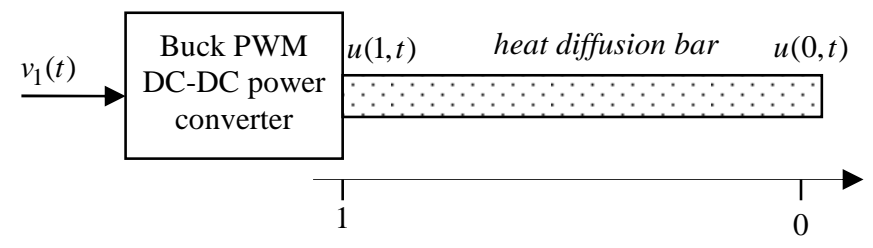

Fig. 1. Example illustrating the system (21)-(24).

\section{B. Observer design and analysis}

Inspired by [6], the following backstepping transformation is considered, for $(x, t) \in[0,1] \times[0,+\infty)$ :

$p(x, t)=u(x, t)-C M(x) M^{-1}(1) X(t)$,

where $M(x) \in \mathbf{R}^{n \times n}$ undergoes the following ODE equation:

$$
\frac{d^{2} M}{d x^{2}}(x)=M(x) A, M(0)=I, \frac{d M}{d x}(0)=0
$$

The matrix gain $M(x)$ plays an instrumental role in the considered observer. For convenience, some of its properties are provided in Appendix B. Using (27), (21) and (22), it follows that the new state $p(x, t)$ defined by (26) undergoes the following PDE, for $(x, t) \in(0,1) \times(0,+\infty)$ a.e.:

$$
p_{t}(x, t)=p_{x x}(x, t)+g(x, v(t))-C M(x) M^{-1}(1) B v(t),
$$

For convenience, the new system representation expressed in terms of the states $(X(t), p(x, t))$ is rewritten, for all $(x, t) \in(0,1) \times(0,+\infty)$ a.e.:

$$
\begin{aligned}
& \dot{X}(t)=A X(t)+B v(t), \\
& p_{t}(x, t)=p_{x x}(x, t)+g(x, v(t))-C M(x) M^{-1}(1) B v(t), \\
& p_{x}(0, t)=p(1, t)=0, \text { for all } t \geq 0 \\
& u(x, t)=p(x, t)+C M(x) M^{-1}(1) X(t),
\end{aligned}
$$

where the boundary conditions (31) are immediately obtained from (26) using (23), (24) and (27). Equations (29)-(32) is an equivalent representation of the initial system model (21)-(24). A key feature of the new representation is that the infinitedimensional subsystem, here defined by (30)-(31), is decoupled from the finite-dimensional subsystem described by (29) (while a coupling existed in the initial model (21)-(24)). This decoupling will prove to be useful to make easier the observer design and analysis. The well-posedness of (22)-(24) is also better analysed based on (30)-(31). This is first made precise in the following Remark:

Remark 4. The well-posedness of the initial value problem (30)(31) can be analyzed using e.g. Theorem 3.1 in [8]. To this end, introduce the set

$$
P=\left\{f \in C^{2}([0,1] ; \mathbf{R}): f^{\prime}(0)=f(1)=0\right\}
$$

and let equation (30) be rewritten in the more compact form,

$$
p_{t}(x, t)=p_{x x}(x, t)+\chi(x, t)
$$

with $\chi(x, t) \stackrel{\text { def }}{=} g(x, v(t))-C M(x) M^{-1}(1) B v(t)$ is acting as an input of (48). By assumption, we have $g \in C^{1}\left([0,1] \times \mathbf{R}^{m} ; \mathbf{R}\right)$ and $v \in C^{1}\left(\mathbf{R}_{+} ; \mathbf{R}^{m}\right)$ and, by Appendix B, $M \in C^{\infty}\left([0,1] ; \mathbf{R}^{n \times n}\right)$. It readily follows that $\chi \in C^{1}\left([0,1] \times \mathbf{R}_{+} ; \mathbf{R}\right)$. It turns out that equation (48) is a particular case of equation (2.6) in [8] and the boundary conditions (31) are also particular cases of (2.7) in [8]. Then, applying Theorem 3.1 in [8] it follows that the evolution equation (48) with (31) and initial condition $p[0] \in P$ has a unique solution $p \in C^{0}\left([0,1] \times \mathbf{R}_{+} ; \mathbf{R}\right) \cap C^{1}([0,1] \times(0,+\infty) ; \mathbf{R})$ for which $p[t] \in P$ for all $t \geq 0$. This result also applies to $u(x, t)=p(x, t)+C M(x) M^{-1}(1) X(t)$, using $(21)$ and the fact that $g \in C^{1}\left([0,1] \times \mathbf{R}^{m} ; \mathbf{R}\right)$ and $M \in C^{\infty}\left([0,1] ; \mathbf{R}^{n \times n}\right) . \triangleleft$

We now focus ourselves on the observer design and analysis. To this end, we start with the following sampled-output observer structure for the system (29)-(32):

$$
\begin{aligned}
\dot{\hat{X}}(t)=A \hat{X}(t)+B v(t)-M(1) L\left(\hat{y}\left(t_{k}\right)-y\left(t_{k}\right)\right), & \\
& \text { for all } t \in\left[t_{k}, t_{k+1}\right) \text { and all integers } k \geq 0 \\
\hat{p}_{t}(x, t)= & \hat{p}_{x x}(x, t)+g(x, v(t))-C M(x) M^{-1}(1) B v(t), \\
& \text { for }(x, t) \in(0,1) \times(0,+\infty) \text { a.e. } \\
\hat{p}_{x}(0, t)= & \hat{p}(1, t)=0, \text { for all } t \geq 0 \\
\hat{u}(x, t)= & \hat{p}(x, t)+C M(x) M^{-1}(1) \hat{X}(t), \\
& \text { for }(x, t) \in[0,1] \times[0,+\infty)
\end{aligned}
$$

with $\hat{y}\left(t_{k}\right)=\hat{u}\left(0, t_{k}\right)$, where $L \in \mathbf{R}^{n}$ is arbitrary vector such that $A-L C$ is a Hurwitz matrix. The last requirement is not an issue since the pair $(A, C)$ is observable. In fact, the observer is a copy of the system (29)-(32) with an additional innovation term in equation (33). To analyse this observer, the following state estimation errors are introduced:

$$
\begin{aligned}
& \tilde{X}(t)=\hat{X}(t)-X(t), \tilde{p}(x, t)=\hat{p}(x, t)-p(x, t) \\
& \tilde{u}(x, t)=\hat{u}(x, t)-u(x, t)
\end{aligned}
$$

Then, using (29)-(32) and (33)-(36), one gets the following error system:

$$
\begin{gathered}
\dot{\tilde{X}}(t)=A \tilde{X}(t)-M(1) L C M^{-1}(1) \tilde{X}\left(t_{k}\right)-M(1) L \tilde{p}\left(0, t_{k}\right), \\
\quad \text { for all } t \in\left[t_{k}, t_{k+1}\right) \text { and all integers } k \geq 0 \\
\tilde{p}_{t}(x, t)=\tilde{p}_{x x}(x, t), \text { for }(x, t) \in(0,1) \times(0,+\infty) \text { a.e. } \\
\tilde{p}_{x}(0, t)=\tilde{p}(1, t)=0, \text { for all } t \geq 0 \\
\tilde{u}(x, t)=\tilde{p}(x, t)+C M(x) M^{-1}(1) \tilde{X}(t), \\
\quad \text { for all }(x, t) \in[0,1] \times[0,+\infty)
\end{gathered}
$$

where the first equation is obtained using the fact that $\hat{y}\left(t_{k}\right)-y\left(t_{k}\right)=\tilde{u}\left(0, t_{k}\right)$ and equations (33) and (36). It is readily seen that, if $(X, w)$ is substituted to $(\tilde{X}, \tilde{p})$ then, the error system (39)-(42) fits the form of the hybrid system (1)-(3) with $A_{0}=A, \quad A_{1}=-M(1) L C M^{-1}(1), \quad b=-M(1) L \quad$ and $\quad z(t)=0$. Then, Proposition 1 can be invoked to analyze the former, provided that $A+A_{1}$ is Hurwitz. This actually is the case because $A-L C$ is Hurwitz and $A_{0}+A_{1}=A-M(1) L C M^{-1}(1)=M(1)(A-L C) M^{-1}(1)$, using the fact that $M(1) A M^{-1}(1)=A$ (due to Property 3 of Appendix B). Then, Proposition 1 can be applied to the error system (39)-(42). Doing so, one gets the following result:

Theorem 1. Consider the system (21)-(24) and the observer (33)-(36) where the gain $L \in \mathbf{R}^{n}$ is selected so that the matrix $A-L C \in \mathbf{R}^{n \times n}$ is Hurwitz. Then, there exist real constants $T, \rho, \sigma>0$ such that, for any $T$-diameter partition $\left\{t_{k}\right\}_{k=0}^{\infty}$, $v \in C^{1}\left(\mathbf{R}_{+} ; \mathbf{R}^{m}\right), \quad X_{0}, \hat{X}_{0} \in \mathbf{R}^{n}, \quad u[0], \hat{p}[0] \in C^{2}[0,1], \quad$ with $(\hat{p}[0])(1)=\left(\hat{p}_{x}[0]\right)(0)=0, \quad\left(u_{x}[0]\right)(0)=0,(u[0])(1)=C X_{0}$, the initial value problem defined by (21)-(24) and (33)-(36) with 
initial conditions $X(0)=X_{0}, \quad \hat{X}(0)=\hat{X}_{0}, u(x, 0)=(u[0])(x)$, $\hat{p}(x, 0)=(\hat{p}[0])(x)$ for $x \in[0,1]$, has a unique solution that satisfies, for all $t \geq 0$ :

$$
|\tilde{X}(t)|+\|\tilde{u}[t]\|+\left\|\tilde{u}_{x}[t]\right\| \leq \rho \exp (-\sigma t)\left(\left|\hat{X}_{0}-X_{0}\right|+\left\|\tilde{p}_{x}[0]\right\|\right)
$$

where $\tilde{X}(t), \tilde{u}[t] \in C^{2}[0,1]$ and $\tilde{p}[t] \in C^{2}[0,1]$ (with $t \geq 0$ ) are defined by (37)-(38).

Proof. It has already been pointed out that Proposition 1 is applicable to the system (39)-(40). Accordingly, for any $\sigma \in(0, \lambda) \cap\left(0, \pi^{2} / 4\right]$ and any $T>0$ sufficiently small so that (9) is satisfied, there exists a constant $K>0$ such that (17) and (7) hold, replacing there $(X, w)$ by $(\tilde{X}, \tilde{p})$ and letting $z(t)=0$. Specifically, one gets the following inequalities:

$$
\begin{gathered}
\|\tilde{p}[t]\| \leq \exp \left(-\frac{\pi^{2}}{4} t\right)\|\tilde{p}[0]\|,\left\|\tilde{p}_{x}[t]\right\| \leq \exp \left(-\frac{\pi^{2}}{4} t\right)\left\|\tilde{p}_{x}[0]\right\| \\
|\tilde{X}(t)| \leq K \exp (-\sigma)\left(|\tilde{X}(0)|+\left\|\tilde{p}_{x}[0]\right\|\right)
\end{gathered}
$$

for all $t \geq 0$. Due to (41) one has, applying Wirtinger's inequality, $\|\tilde{p}[t]\| \leq\left\|\tilde{p}_{x}[t]\right\|$, for all $t \geq 0$. This together with (42) and (37)-(38) yield, for all $t \geq 0$ :

$$
\begin{aligned}
\|\tilde{u}[t]\| & \leq\|\tilde{p}[t]\|+\max _{0 \leq x \leq 1}\left(\left|C M(x) M^{-1}(1)\right|\right)|\tilde{X}(t)| \\
& \leq\left\|\tilde{p}_{x}[t]\right\|+\max _{0 \leq x \leq 1}\left(\left|C M(x) M^{-1}(1)\right|\right)|\tilde{X}(t)| \\
\left\|\tilde{u}_{x}[t]\right\| & \leq\left\|\tilde{p}_{x}[t]\right\|+\max _{0 \leq x \leq 1}\left(\left|C \frac{d M}{d x}(x) M^{-1}(1)\right||| \tilde{X}(t) \mid\right.
\end{aligned}
$$

It follows from (44)-(47) that there is a $\rho>0$ such that,

$$
|\tilde{X}(t)|+\|\tilde{u}[t]\|+\left\|\tilde{u}_{x}[t]\right\| \leq \rho \exp (-\sigma)\left(|\tilde{X}(0)|+\left\|\tilde{p}_{x}[0]\right\|\right),
$$

for $t \geq 0$, which proves (43) and establishes Theorem 1 . $\triangleleft$

Remark 5. Estimates of the constants $\sigma, T>0$ in Theorem 1 can be obtained this way: first, by Proposition 1, $\sigma \in(0, \lambda) \cap\left(0, \pi^{2} / 4\right]$ with $\lambda$ any real constant such that

$$
\left|\exp \left(\left(A+A_{1}\right) t\right)\right|=\left|M(1) \exp ((A-L C) t) M^{-1}(1)\right| \leq R \exp (-\lambda t),
$$

for all $t \geq 0$, with $A_{1}=-M(1) L C M^{-1}(1)$. Then, $T>0$ is selected so that (9) holds where $\phi: \mathbf{R}_{+} \rightarrow \mathbf{R}_{+}$is any continuous function satisfying $|\exp (A t)| \leq \phi(t)$, for all $t \geq 0$. However, it should be noticed that inequality (9) provides a conservative upper bound for the diameter $T>0$ of the sampling partition $\left\{t_{k}\right\}_{k=0}^{\infty}$. That is, in practice, the observer (33)-(36) works well even with some values of $T>0$ not satisfying inequality (9).

Remark 6. Notice that the exponential convergence (43) holds for every sampling partition $\left\{t_{k}\right\}_{k=0}^{\infty}$ with diameter $T>0$. It turns out that performance (43) is robust to sampling schedule. For example, if the measurement device is set to provide measurements every $T / m$ time units, for some positive integer $m$, it is guaranteed that the performance (43) will be preserved even if $m-1$ consecutive measurements are periodically lost.

\section{APPROXIMATE SAMPLED-OUTPUT OBSERVER FOR ODE-PDE CASCADES}

The practical difficulty with the observer (33)-(36) is that its realtime implementation necessitates an online numerical solution of a PDE, i.e. (34)-(35). This will now be coped with using a finite- dimensional approximation of PDE (34)-(35), based on eigenfunction expansion. First, it is checked that the feedback term in (33) is expressed, in terms of $\hat{p}(0, t)$, as follows:

$M(1) L\left(\hat{y}\left(t_{k}\right)-y\left(t_{k}\right)\right)=M(1) L\left(\hat{p}\left(0, t_{k}\right)+C M^{-1}(1) \hat{X}\left(t_{k}\right)-y\left(t_{k}\right)\right)$ where we have used (36), (27) and the fact that $\hat{y}\left(t_{k}\right)=\hat{u}\left(0, t_{k}\right)$. Then, (33) rewrites as follows:

$$
\begin{aligned}
\dot{\hat{X}}(t)= & A \hat{X}(t)+B v(t) \\
& -M(1) L\left(\hat{p}\left(0, t_{k}\right)+C M^{-1}(1) \hat{X}\left(t_{k}\right)-y\left(t_{k}\right)\right)
\end{aligned}
$$

for all $t \in\left[t_{k}, t_{k+1}\right)$ and all integers $k \geq 0$. Then, the following approximate observer, inspired by the exact observer described by (33)-(36) and (49), is considered:

$$
\begin{aligned}
\dot{\bar{X}}(t)= & A \bar{X}(t)+B v(t) \\
& -M(1) L\left(\bar{p}\left(0, t_{k}\right)+C M^{-1}(1) \bar{X}\left(t_{k}\right)-y\left(t_{k}\right)\right) \\
& \text { for all } t \in\left[t_{k}, t_{k+1}\right) \text { and all integers } k \geq 0 \\
\bar{p}(x, t) \stackrel{\text { def }}{=} & \sqrt{2} \sum_{l=0}^{N} c_{l}(t) \cos \left((2 l+1) \frac{\pi x}{2}\right) \\
\dot{c}_{l}(t)= & -(2 l+1)^{2} \frac{\pi^{2}}{4} c_{l}(t) \\
& +\sqrt{2} \int_{0}^{1} g_{a}(x, v(t)) \cos \left((2 l+1) \frac{\pi x}{2}\right) d x \\
& \text { def } \\
\bar{u}(x, t)= & \bar{p}(x, t)+C M(x) M^{-1}(1) \bar{X}(t)
\end{aligned}
$$

for all $(x, t) \in[0,1] \times[0,+\infty)$ and $l=0,1,2, \ldots, N$, with

$$
g_{a}(x, v)=g(x, v)-C M(x) M^{-1}(1) v, \text { for }(x, v) \in[0,1] \times \mathbf{R}^{m}
$$

The following theorem states that system (50)-(52) is an approximate exponential observer for system (21)-(24), provided that the sampling period is sufficiently small.

Theorem 2. Consider the system (21)-(24) and the approximate observer, described by (50)-(52) with $L \in \mathbf{R}^{n}$ is such that $A-L C \in \mathbf{R}^{n \times n}$ is Hurwitz. Suppose that there exists a constant $P_{g}>0$ such that $|g(x, v)| \leq P_{g}|v|$ for all $(x, v) \in[0,1] \times \mathbf{R}^{m}$. Then, there exist (sufficiently small) constants $\sigma, T>0$ and (sufficiently large, $N$-independent) constants $\rho, \beta>0$ such that, for any $T$-diameter partition $\left\{t_{k}\right\}_{k=0}^{\infty}$ of $\mathbf{R}_{+}$, any bounded $v \in C^{1}\left(\mathbf{R}_{+} ; \mathbf{R}^{m}\right), \quad X_{0}, \bar{X}_{0} \in \mathbf{R}^{n}, \quad c(0) \in \mathbf{R}^{N+1}, \quad u[0] \in C^{2}[0,1]$ with $\left(u_{x}[0]\right)(0)=0$ and $(u[0])(1)=C X_{0}$, the initial value problem defined by (29)-(32) and (50)-(54) with initial conditions $\left(c_{0}(0), \ldots, c_{N}(0)\right)=c(0), \quad X(0)=X_{0}, \quad \bar{X}(0)=\bar{X}_{0}$, $u(x, 0)=(u[0])(x)$ for $x \in[0,1]$, has a unique solution satisfying:

$$
\begin{aligned}
\mid \bar{X}(t) & -X(t) \mid+\max _{0 \leq x \leq 1}(|\bar{u}(x, t)-u(x, t)|) \\
& \leq \rho \exp (-\sigma)\left(\left|\bar{X}_{0}-X_{0}\right|+\left\|\varphi_{x}\right\|\right)+\frac{\beta}{N+1} \sup _{s \geq 0}(|v(s)|)
\end{aligned}
$$

for all $t \geq 0$, where

$$
\begin{aligned}
\varphi(x) \stackrel{\text { def }}{=} & \sqrt{2} \sum_{l=0}^{N} c_{l}(0) \cos \left((2 l+1) \frac{\pi x}{2}\right) \\
& -(u[0])(x)+C M(x) M^{-1}(1) X_{0}, \text { for } x \in[0,1] .
\end{aligned}
$$

Proof. Consider the system,

$$
\begin{gathered}
q_{t}(x, t)=q_{x x}(x, t)+g(x, v(t))-C M(x) M^{-1}(1) B v(t), \\
\quad \text { for all }(x, t) \in(0,1) \times(0,+\infty), \\
q_{x}(0, t)=q(1, t)=0, \text { for all } t \geq 0
\end{gathered}
$$


with initial condition

$$
(q[0])(x)=\sqrt{2} \sum_{l=0}^{N} c_{l}(0) \cos \left((2 l+1) \frac{\pi x}{2}\right), \text { for } x \in[0,1]
$$

Its solution writes as follows, for $(x, t) \in[0,1] \times \mathbf{R}_{+}$:

$q(x, t)=\sqrt{2} \sum_{l=0}^{\infty} c_{l}(t) \cos \left((2 l+1) \frac{\pi x}{2}\right)$,

where $\quad c_{l}(0)=\sqrt{2} \int_{0}^{1}(q[0])(x) \cos \left((2 l+1) \frac{\pi x}{2}\right) d x \quad$ while $\quad c_{l}(t)$ $(l=0,1, \ldots)$ satisfy $(52)$. We conclude from $(52)$ (for $l=N+1, \ldots)$ and (58) (which entails $c_{l}(0)=0$ for $l=N+1, \ldots$ ) that the following equations hold, for $l=N+1, \ldots$ and all $t \geq 0$ :

$$
\begin{aligned}
c_{l}(t)=\sqrt{2} \int_{0}^{t} & \exp \left(-(2 l+1)^{2} \frac{\pi^{2}}{4}(t-s)\right) \\
& \times\left(\int_{0}^{1} g_{a}(z, v(s)) \cos \left((2 l+1) \frac{\pi z}{2}\right) d z\right) d s
\end{aligned}
$$

Also, one immediately gets comparing (60) and (51):

$$
q(0, t)-\bar{p}(0, t)=\sqrt{2} \sum_{l=N+1}^{\infty} c_{l}(t)
$$

Next, we define for $(x, t) \in[0,1] \times \mathbf{R}_{+}$:

$$
\begin{aligned}
& e(t)=\bar{X}(t)-X(t) \\
& w(x, t)=q(x, t)-p(x, t)
\end{aligned}
$$

Using (63), (56), (57), (30), (31), it follows that equations (2), (3) hold. Subtracting both sides of (29) from corresponding sides of (50), it successively follows, for all $t \in\left[t_{k}, t_{k+1}\right)$ and all $k \geq 0$ :

$$
\begin{aligned}
\dot{e}(t)= & A e(t)-M(1) L\left(\bar{p}\left(0, t_{k}\right)+C M^{-1}(1) \bar{X}\left(t_{k}\right)-u\left(0, t_{k}\right)\right) \\
= & A e(t)-M(1) L C M^{-1}(1) e\left(t_{k}\right) \\
& \left.+M(1) L\left(p\left(0, t_{k}\right)-\bar{p}\left(0, t_{k}\right)\right) \text { (using }(26)\right) \\
= & A e(t)-M(1) L C M^{-1}(1) e\left(t_{k}\right) \\
& -M(1) L w\left(0, t_{k}\right)+M(1) L \sqrt{2} \sum_{l=N+1}^{\infty} c_{l}\left(t_{k}\right)
\end{aligned}
$$

where the last equality is obtained using (63) and (61). It follows that equations (1)-(3) hold with $X(t)$ being replaced by $e(t)$ and $z(t)=\sqrt{2} \sum_{l=N+1}^{\infty} c_{l}\left(t_{k}\right) \quad\left(\right.$ for $t \in\left[t_{k}, t_{k+1}\right)$ and all integers $\left.k \geq 0\right)$, $A_{0}=A, \quad A_{1}=-M(1) L C M^{-1}(1), \quad b=-M(1) L, \quad G=M(1) L$. Applying Proposition 1, it follows that for any (sufficiently small) $\sigma, T>0$ there exist constants $K, \gamma>0$ such that (7) holds as well as the following estimate, for all $t \geq 0$ :

$$
|e(t)| \leq K \exp (-\sigma)\left(|e(0)|+\| w_{x}[0]||\right)+\gamma \sup _{0 \leq s \leq t}\left|\sqrt{2} \sum_{l=N+1}^{\infty} c_{l}(s)\right|(65)
$$

On the other hand, definition (54) in conjunction with the fact that $|g(x, v)| \leq P_{g}|v|$, for all $(x, v) \in[0,1] \times \mathbf{R}^{m}$, and Property 4 in Appendix B imply that there exists a constant $P_{a}>0$ (independent of $v \in C^{1}\left(\mathbf{R}_{+} ; \mathbf{R}^{m}\right)$ ) such that:

$$
\left|g_{a}(x, v(t))\right| \leq P_{a} \sup _{s \geq 0}(|v(s)|) \text {, for all }(x, t) \in[0,1] \times \mathbf{R}_{+}
$$

Then, it follows from (60) and (66) that the following inequalities hold, for all $t \geq 0$ :

$\left|c_{l}(t)\right| \leq \frac{4 \sqrt{2}}{(2 l+1)^{2} \pi^{2}} P_{a} \sup _{s \geq 0}(|v(s)|)$, with $l=N+1, N+2 \ldots$
It is readily checked that:

$$
\sum_{l=N+1}^{\infty} \frac{1}{(2 l+1)^{2}} \leq \sum_{k=2 N+3}^{\infty} \frac{1}{k^{2}} \leq \int_{2 N+2}^{\infty} \frac{1}{x^{2}} d x=\frac{1}{2 N+2}
$$

Then, it follows from (67) and (68) that:

$$
\begin{aligned}
\sup _{s \geq 0}\left|\sqrt{2} \sum_{l=N+1}^{\infty} c_{l}(s)\right| \leq & \frac{8 P_{a}}{\pi^{2}}\left(\sum_{l=N+1}^{\infty} \frac{1}{(2 l+1)^{2}}\right) \sup _{s \geq 0}(|v(s)|) \\
& \leq \frac{4 P_{a}}{\pi^{2}(N+1)} \sup _{s \geq 0}(|v(s)|)
\end{aligned}
$$

Using (69), one gets from (65) that, for all $t \geq 0$ :

$|e(t)| \leq K \exp (-\sigma)\left(|e(0)|+\left\|w_{x}[0]\right\|\right)+\frac{4 P_{a}}{\pi^{2}(N+1)} \sup _{s \geq 0}(|v(s)|)$

On the other hand, it follows from (53), (59), (62) and (63) that, for all $(x, t) \in[0,1] \times \mathbf{R}_{+}$:

$$
\begin{aligned}
\bar{u}(x, t)-u(x, t) & =\bar{p}(x, t)-q(x, t)+w(x, t)+C M(x) M^{-1}(1) e(t) \\
& =w(x, t)+C M(x) M^{-1}(1) e(t) \\
& -\sqrt{2} \sum_{l=N+1}^{\infty} c_{l}(t) \cos \left((2 l+1) \frac{\pi x}{2}\right)
\end{aligned}
$$

where the last equality is obtained using (51) and (59). It follows from (71) that:

$$
\max _{0 \leq x \leq 1}|\bar{u}(x, t)-u(x, t)| \leq \max _{0 \leq x \leq 1}|w(x, t)|+\mu|e(t)|+\sqrt{2} \sum_{l=N+1}^{\infty}\left|c_{l}(t)\right|
$$

where $\mu=\max _{0 \leq x \leq 1}\left|C M(x) M^{-1}(1)\right|$ exists by Property 4 in Appendix B. In view of (3), one has $w(x, t)=-\int_{0}^{x} w_{x}(s, t) d s$, for all $t \geq 0$ and $x \in[0,1]$. Using the Cauchy-Schwarz inequality, one gets for all $t \geq 0$ and $x \in[0,1]$ :

$$
\begin{aligned}
|w(x, t)| & =\left|\int_{0}^{x} w_{x}(s, t) d s\right| \leq \int_{0}^{x}\left|w_{x}(s, t)\right| d s \\
& \leq \sqrt{x} \sqrt{\int_{0}^{x}\left|w_{x}(s, t)\right|^{2} d s} \leq\left\|w_{x}[t]\right\|
\end{aligned}
$$

This, combined with the second inequality in (7), gives $\max _{0 \leq x \leq 1}|w(x, t)| \leq \exp \left(-\frac{\pi^{2}}{4} t\right)\left\|w_{x}[0]\right\| \leq \exp (-\sigma)\left\|w_{x}[0]\right\|, \quad$ for $\quad$ all $t \geq 0$, using the fact that $\sigma \leq \pi^{2} / 4$ (by Proposition 1). Then, it follows from (72), (70), (69) and (67), that

$$
\begin{aligned}
\max _{0 \leq x \leq 1}|\bar{u}(x, t)-u(x, t)| \\
\leq \exp (-\sigma)|| w_{x}[0] \mid+K \mu \exp (-\sigma)\left(|e(0)|+\left\|w_{x}[0]\right\|\right) \\
\quad+\frac{4 \gamma \mu P_{a}}{\pi^{2}(N+1)} \sup _{s \geq 0}(|v(s)|)+\frac{4 \gamma P_{a}}{\pi^{2}(N+1)} \sup _{s \geq 0}(|v(s)|)
\end{aligned}
$$

Combining (73) and (70) gives (55), for some constants $\rho, \beta>0$, and completes the proof of Theorem 2 . $\triangleleft$

Remark 7. Theorem 2 shows that the accuracy of the approximate estimates $\bar{X}(t)$ and $\bar{u}(x, t)$ depends on the truncation order $N$ in (51) (compared to (59)). The larger $N$ the better the estimate accuracy. However, a large $N$ entails an increase of computational load. Practically, a trade-off between estimate accuracy and computational load is made. 


\section{ILLUSTRATION EXAMPLE}

Practical use of Theorems 1 and 2 entails the computation of the solution of equations (27) and the choice of the MASP $T$. Consider the following system of the form (21)-(24):

$$
\begin{aligned}
& \dot{X}_{1}(t)=X_{2}(t), \dot{X}_{2}(t)=v_{1}(t) \\
& u_{t}(x, t)=u_{x x}(x, t)+\psi(x) v_{2}(t), \\
& u_{x}(0, t)=0 \text { and } u(1, t)=X_{1}(t), \text { for all } t \geq 0
\end{aligned}
$$

with the output signal:

$$
y(t)=u\left(0, t_{k}\right) \text {, for all } t \in\left[t_{k}, t_{k+1}\right) \text { and all } k \geq 0
$$

where $X=\left[X_{1}, X_{2}\right]^{T} \in \mathbf{R}^{2} \quad$ denotes a state vector, $v=\left[v_{1}, v_{2}\right]^{T} \in C^{1}\left(\mathbf{R}_{+} ; \mathbf{R}^{2}\right)$ is an input vector, $\psi \in C^{1}([0,1] ; \mathbf{R})$ is a given function, and $\left\{t_{k}\right\}_{k=0}^{\infty}$ is a $T$-diameter partition of $\mathbf{R}_{+}$. Clearly, the system (74)-(77) fits the form (21)- (24) with

$$
A=\left[\begin{array}{ll}
0 & 1 \\
0 & 0
\end{array}\right], B=\left[\begin{array}{ll}
0 & 0 \\
1 & 0
\end{array}\right], C=\left[\begin{array}{ll}
1 & 0
\end{array}\right], g(x, v)=\psi(x) v_{2}
$$

It is checked that the solution of equations (27) is:

$$
M(x)=\left[\begin{array}{cc}
1 & x^{2} / 2 \\
0 & 1
\end{array}\right]
$$

By Theorem 1, an exponential observer for system (74)-(77) is:

$$
\left\{\begin{array}{l}
\dot{\hat{X}}_{1}=\hat{X}_{2}-\left(l_{1}+\frac{1}{2} l_{2}\right)\left(\hat{p}\left(0, t_{k}\right)+\hat{X}_{1}\left(t_{k}\right)-\frac{1}{2} \hat{X}_{2}\left(t_{k}\right)-y\left(t_{k}\right)\right) \\
\dot{\hat{X}}_{2}=v_{1}(t)-l_{2}\left(\hat{p}\left(t_{k}, 0\right)+\hat{X}_{1}\left(t_{k}\right)-\frac{1}{2} \hat{X}_{2}\left(t_{k}\right)-y\left(t_{k}\right)\right)
\end{array}\right.
$$

$$
\begin{gathered}
\hat{p}_{t}(x, t)=\hat{p}_{x x}(x, t)+\psi(x) v_{2}(t)+\frac{1-x^{2}}{2} v_{1}(t), \\
\quad \text { for }(x, t) \in(0,1) \times(0,+\infty) \\
\hat{p}_{x}(x, t)=\hat{p}(1, t)=0, \text { for all } t \geq 0 \\
\hat{u}(x, t)=\hat{p}(x, t)+\hat{X}_{1}(t)+\frac{x^{2}-1}{2} \hat{X}_{2}(t), \\
\text { for }(x, t) \in[0,1] \times[0,+\infty)
\end{gathered}
$$

whatever $l_{1}, l_{2}>0$, and sufficiently small MASP $T>0$. Proposition 1 and Remark 5 are resorted to estimate $T$ and $\sigma>0$ so that $\sup _{t>0}(\exp (\sigma)|X(t)-\hat{X}(t)|)<+\infty$. To this end, direct computations give:

$$
\begin{aligned}
& A_{0}=\left[\begin{array}{ll}
0 & 1 \\
0 & 0
\end{array}\right], \quad A_{1}=-M(1)\left[\begin{array}{ll}
l_{1} & 0 \\
l_{2} & 0
\end{array}\right] M^{-1}(1), \\
& \exp \left(\left(A_{0}+A_{1}\right) t\right)=M(1) \exp \left(\left[\begin{array}{ll}
-l_{1} & 1 \\
-l_{2} & 0
\end{array}\right] t\right) M^{-1}(1) \\
& \exp \left(A_{0} t\right)=\left[\begin{array}{ll}
1 & t \\
0 & 1
\end{array}\right], \quad\left|\exp \left(A_{0} t\right)\right| \leq \phi(t)=t+1
\end{aligned}
$$

Letting $l_{1}=l_{2}=2$, one finds that,

$$
\left|\exp \left(\left[\begin{array}{ll}
-l_{1} & 1 \\
-l_{2} & 0
\end{array}\right]\right)\right| \leq(1+\sqrt{5}) \exp (-t) \text {, for all } t \geq 0 \text {. }
$$

Then, with the notations of Theorem 1, one gets the estimates:

$$
\begin{aligned}
& \lambda=1,\left|A_{0}\right|=1,\left|A_{1}\right|=\frac{\sqrt{65}}{2},|M(1)|=\left|M^{-1}(1)\right|=\sqrt{\frac{9+\sqrt{17}}{8}} \\
& \left|\exp \left(\left(A_{0}+A_{1}\right) t\right)\right| \leq \frac{9+\sqrt{17}}{8}(1+\sqrt{5}) \exp (-t)
\end{aligned}
$$

Then, inequality (9) yields:

$$
1>\sigma+107.67 \exp (\sigma T)\left(T+\frac{T^{2}}{2}\right)
$$

This implies that $1>\sigma+107.67 T$ and so $T<0.00924$. Setting the value of the sampling period to $T=0.001$, one gets from (84) that $\sigma<0.892, \quad$ indicating that $\sup _{t>0}(\exp (\sigma t)|X(t)-\hat{X}(t)|)<+\infty$ for all $\sigma<0.892$. Now, we know by Theorem 2 that if the amplitude of the input vector $v=\left[v_{1}, v_{2}\right]^{T} \in C^{1}\left(\mathbf{R}_{+} ; \mathbf{R}^{2}\right)$ is not large then, the following set of equations defines an approximate exponential observer for system (74)-(76) provided that $T<0.00924$ :

$$
\begin{gathered}
\dot{\bar{X}}_{1}(t)=\bar{X}_{2}(t)-3\left(\sqrt{2} \sum_{l=0}^{N} c_{l}\left(t_{k}\right)+\bar{X}_{1}\left(t_{k}\right)-\frac{1}{2} X_{2}\left(t_{k}\right)-y\left(t_{k}\right)\right) \\
\dot{\bar{X}}_{2}(t)=v_{1}(t)-2\left(\sqrt{2} \sum_{l=0}^{N} c_{l}\left(t_{k}\right)+\bar{X}_{1}\left(t_{k}\right)-\frac{1}{2} \bar{X}_{2}\left(t_{k}\right)-y\left(t_{k}\right)\right) \\
\dot{c}_{l}(t)=-(2 l+1)^{2} \frac{\pi^{2}}{4} c_{l}(t)+v_{2}(t) \sqrt{2} \int_{0}^{1} \psi(x) \cos \left((2 l+1) \frac{\pi x}{2}\right) d x \\
+\frac{\sqrt{2}}{2} v_{1}(t) \int_{0}^{1}\left(1-x^{2}\right) \cos \left((2 l+1) \frac{\pi x}{2}\right) d x, \text { for } l=0,1, \ldots, N \\
\bar{u}(x, t)=\bar{X}_{1}(t)+\left(x^{2}-1\right) \bar{X}_{2}(t) / 2+\sqrt{2} \sum_{l=0}^{N} c_{l}(t) \cos \left((2 l+1) \frac{\pi x}{2}\right), \\
\text { for all }(x, t) \in[0,1] \times[0,+\infty)
\end{gathered}
$$

\section{CONCLUSION}

The contribution of this work is threefold. First, the exponential stability result of Proposition 2 is established for hybrid systems composed of an ODE and a PDE interacting according to equations (1)-(4). Then, the problem of sampled-output observer design for the ODE-PDE cascade (19)-(22) is dealt with using the backstepping-like transformation (24). The obtained observer (33)-(36) is shown in Theorem 1 to be exponentially stable. Finally, the practical observer (48)-(52) that includes no PDEs is derived and shown in Theorem 2 to be a suitable approximation of (33)-(36). Proposition 1 plays an instrumental role in the proofs of Theorem 1 and Theorem 2 because both result in an observation error system fitting the hybrid system structure (1)(4). As a matter of fact, Proposition 1 can as well constitute a suitable framework for sampled-data output-feedback control of linear ODE-PDE cascades. This perspective is currently under investigation.

\section{APPENDICES}

Appendix A. Proof of Lemma 1.

The solution of (2)-(3) is well-defined satisfying, for all $t>0$ :

$$
\begin{aligned}
w(x, t)= & 2 \sum_{l=0}^{\infty} \cos \left((2 l+1) \frac{\pi x}{2}\right) \exp \left(-\frac{(2 l+1)^{2} \pi^{2}}{4} t\right) \\
& \times \int_{0}^{1}(w[0])(s) \cos \left((2 l+1) \frac{\pi s}{2}\right) d s
\end{aligned}
$$

To show inequalities (7), notice first that $\left\{\phi_{l}(x)=\sqrt{2} \cos \left((2 l+1) \frac{\pi x}{2}\right)\right\}_{l=0}^{\infty}$ is an orthonormal basis of $L^{2}(0,1)$ (they are the eigenfunctions of the Sturm-Liouville 
operator

$$
d^{2} / d x^{2}
$$
defined

$\left.\Omega=\left\{f \in C^{2}([0,1] ; \mathbf{R}): f(1)=\frac{d f}{d x}(0)=0\right\}\right) . \quad$ Then, $\quad$ applying

Parseval's identity to $w(x, t)$ one gets, using (A1):

$$
\begin{aligned}
\|w[t]\|^{2}=2 \sum_{l=0}^{\infty}\left(\int_{0}^{1}(w[t])(s) \cos \left((2 l+1) \frac{\pi s}{2}\right) d s\right)^{2} \\
\quad=2 \sum_{l=0}^{\infty}\left(\exp \left(-\frac{(2 l+1)^{2} \pi^{2}}{4} t\right) \int_{0}^{1}(w[0])(s) \cos \left((2 l+1) \frac{\pi s}{2}\right) d s\right)^{2} \\
\quad \leq \exp \left(-\frac{\pi^{2}}{2} t\right)\|w[0]\|^{2}
\end{aligned}
$$

which proves the first inequality in (7). On the other hand, differentiating both sides of (A1) with respect to $x$ one gets:

$$
\begin{gathered}
w_{x}(x, t)=-\pi \sum_{l=0}^{\infty}(2 l+1) \sin \left((2 l+1) \frac{\pi x}{2}\right) \exp \left(-\frac{(2 l+1)^{2} \pi^{2}}{4} t\right) \\
\quad \times \int_{0}^{1}(w[0])(s) \cos \left((2 l+1) \frac{\pi s}{2}\right) d s \\
=2 \sum_{l=0}^{\infty} \sin \left((2 l+1) \frac{\pi x}{2}\right) \exp \left(-\frac{(2 l+1)^{2} \pi^{2}}{4} t\right) \\
\quad \times \int_{0}^{1}\left(w_{x}[0]\right)(s) \sin \left((2 l+1) \frac{\pi s}{2}\right) d s, \text { for } t>0
\end{gathered}
$$

where the last equality has been obtained using an integration by parts and the fact that $(w[0])(1)=0$. Note that the functions $\left\{\psi_{l}(x)=\sqrt{2} \sin \left((2 l+1) \frac{\pi x}{2}\right)\right\}_{l=0}^{\infty}$ constitute an orthonormal basis of $L^{2}(0,1)$ (they are the eigenfunctions of the SturmLiouville operator $\frac{d^{2}}{d x^{2}}$ defined on $\left.\Psi=\left\{f \in C^{2}([0,1] ; \mathbf{R}): f(0)=\frac{d f}{d x}(1)=0\right\}\right) . \quad$ Then, $\quad$ applying Parseval's identity to $w_{x}(x, t)$ one gets, using the right side of (6):

$$
\begin{aligned}
& \left\|w_{x}[t]\right\|^{2}=2 \sum_{l=0}^{\infty}\left(\int_{0}^{1}\left(w_{x}[t]\right)(s) \sin \left((2 l+1) \frac{\pi s}{2}\right) d s\right)^{2} \\
& =2 \sum_{l=0}^{\infty}\left(\exp \left(-\frac{(2 l+1)^{2} \pi^{2}}{4} t\right) \int_{0}^{1}\left(w_{x}[0]\right)(s) \sin \left((2 l+1) \frac{\pi s}{2}\right) d s\right)^{2} \\
& \leq 2 \exp \left(-\frac{\pi^{2}}{2} t\right) \sum_{l=0}^{\infty}\left(\int_{0}^{1}\left(w_{x}[0]\right)(s) \cos \left((2 l+1) \frac{\pi s}{2}\right) d s\right)^{2} \\
& \leq \exp \left(-\frac{\pi^{2}}{2} t\right)\left\|w_{x}[0]\right\|^{2}
\end{aligned}
$$

which proves the second inequality (7) for $t>0$.

\section{Appendix B. Additional properties of $M(x)$}

The function $M(x)$ defined by (27) has the following properties, see proof in [14]:

1) $M(x)=\mathbf{I}+\sum_{k=1}^{\infty} \frac{x^{2 k}}{(2 k) !} A^{k}$

2) $M(x) A=A M(x)$
3) $M^{-1}(x) A=A M^{-1}(x)$

4) $M(x) \stackrel{\text { def }}{=}(\mathbf{I} \quad 0) e^{\left(\begin{array}{ll}0 & A \\ \mathbf{I} & 0\end{array}\right)^{x}}\left(\begin{array}{l}\mathbf{I} \\ 0\end{array}\right) \in \mathbf{R}^{n \times n}, \forall x \in \mathbf{R}$

\section{REFERENCES}

[1] Karafyllis I, C. Kravaris. From continuous-time design to sampled-data design of observers. IEEE Trans. on Aut. Control, vol. 54 (9), pp. 2169 2174, 2009.

[2] Ahmed-Ali T., I. Karafyllis, F. Lamnabhi-Lagarrigue. Global exponential sampled-data observers for nonlinear systems with delayed measurements, Systems \& Control Letters, vol. 62 (7), pp. 539-549, 2013.

[3] Folin T., T. Ahmed-Ali, F. Giri, L. Burlion, F. Lamnabhi-Lagarrigue Sampled-data adaptive observer for a class of state-affine output-injection nonlinear systems. IEEE Transactions on Automatic Control, Vol. 61(2), pp. 462-467, 2016.

[4] Fridman E., and A. Blighovsky. Robust sampled-data control of a class of semilinear parabolic systems. Automatica, vol. 48, pp. 826-836, 2012.

[5] Ahmed-Ali T., E. Fridman, F. Giri, L. Burlion, and F. LamnabhiLagarrigue. A new approach to enlarging sampling intervals for some sampled-data systems and observers. To appear in Automatica, 2016.

[6] Krstic M. Compensating actuator and sensor dynamics governed by diffusion PDEs. Systems \& Control Letters, vol. 58, pp. 372-377, 2009.

[7] Zheng S. Nonlinear Evolution Equations. London, U.K.: Chapman \& Hall, 2004.

[8] I. Karafyllis and M. Krstic, "ISS with Respect to Boundary Disturbances for 1-D Parabolic PDEs", IEEE Trans. on Aut. Control. DOI: 10.1109/TAC.2016.2519762. To appear.

[9] Arcak M. and Dragan Nesic. A framework for nonlinear sampled-data observer design via approximate discrete-time models and emulation. Automatica, Vol. 40 (11), pp. 1931-1938, 2004.

[10] Beikzadeh H., and H.J. Marquez, Input-to-Error Stable Observer Design for Nonlinear Sampled-Data Systems with Application to One-Sided Lipschitz Systems. Automatica. Vol. 67, pp. 1-7, 2016.

[11] Dashkovskiy, S., and A. Mironchenko, "Input-to-State Stability of InfiniteDimensional Control Systems", Mathematics of Control, Signals, and Systems, 25(2.1), pp. 1-35, 2013.

[12] Ahmed-Ali T., I. Karafyllis, F. Giri, M. Krstic and F. Lamnabhi-Lagarrigue. Stability Result for a Class of Sampled-Data Systems and Application to Observer Design for Cascade ODE-PDE systems. 22nd International Symposium on Mathematical Theory of Networks and Systems (MTNS 2016), Minneapolis, MN, USA, 2016.

[13] El Fadil H., F. Giri, F.Z. Chaoui. Accounting for Input Limitation in the control of buck power converters. IEEE Transactions on Circuits and Systems I, Vol. 56, No. 6, pp. 1260-1271, 2009.

[14] Ahmed-Ali T., F. Giri, M. Krstic, F. Lamnabhi-Lagarrigue. Observer Design for a Class of Nonlinear ODE-PDE Cascade Systems. Systems \& Control Letters, vol. 83, pp. 19-27, 2015. 